\title{
Generation of spectrally stable 6.5-fs visible pulses via filamentation in krypton
}

\author{
Keisuke Kaneshima, Kengo Takeuchi, Nobuhisa Ishii, and Jiro Itatani \\ The Institute for Solid State Physics, The University of Tokyo, 5-1-5 Kashiwanoha, Kashiwa, Chiba, 277-8581, Japan \\ (Received 21 January 2016; revised 22 May 2016; accepted 25 May 2016)
}

\begin{abstract}
We produced $5-\mu \mathrm{J}, 6.5$-fs visible pulses at a repetition rate of $1 \mathrm{kHz}$ using filamentation in a gas cell filled with krypton followed by spectral selection and phase compensation by a combination of dielectric mirrors. The visible pulses have a smooth spectrum from 520 to $650 \mathrm{~nm}$ with a shot-to-shot stability in each spectral component of better than $2 \%$ (standard deviation). This pulse compression scheme is simple and robust, and can be easily integrated into intense ultrashort-pulse laser systems.
\end{abstract}

Keywords: filamentation; ultrafast spectroscopy; ultrashort visible pulses

\section{Introduction}

Recent progress of optical parametric chirped-pulse amplifiers (OPCPAs) has realized the generation of intense fewcycle pulses in the IR with stable carrier-envelope phases (CEPs). Using such novel sources, the spectral range of high harmonics was extended from the extreme ultraviolet to the soft-X-ray regions ${ }^{[1-3]}$. The CEP dependence of high harmonic cutoffs suggests the generation of attosecond continua that cover the carbon $K$ edge $(\sim 284 \mathrm{eV})^{[2,3]}$. The next challenge is to realize time-resolved soft-x-ray absorption spectroscopy of carbon materials with femtosecond to attosecond time resolution. One of the prototypical carbon materials that exhibits ultrafast electronic dynamics is polydiacetylene that has absorption bands in the visible wavelength $^{[4]}$. Therefore, it is desirable to establish a method for producing few-femtosecond visible pulses with moderate pulse energy $(>1 \mu \mathrm{J})$, that can be easily integrated into ultrafast intense laser sources for high harmonic generation in the soft x-ray.

For producing ultrashort visible pulses, there are several approaches. The first is a beta-barium borate (BBO)-based noncollinear optical parametric amplifier (NOPA) pumped by the second harmonic of Ti:sapphire lasers ${ }^{[5,6]}$. A typical setup of such a NOPA is usually complicated because it adopts several nonlinear processes, i.e., second-harmonic

Correspondence to: K. Kaneshima, The Institute for Solid State Physics, The University of Tokyo, 5-1-5 Kashiwanoha, Kashiwa, Chiba, 277-8581, Japan. Email: kaneshima@issp.u-tokyo.ac.jp generation (SHG), white-light generation and parametric amplification. The second is to use self-phase modulation (SPM) in a gas-filled hollow fiber ${ }^{[7]}$. Although the hollowfiber pulse compression is successfully used for producing intense few-cycle pulses, there are few applications in pumpprobe spectroscopy ${ }^{[8]}$. It is mainly because the output spectra are less smooth than the output from NOPAs. Furthermore, good pointing stability and short pulse duration ( $<30 \mathrm{fs}$ ) of the input pulses are necessary for stable and broad output spectra.

Spectral broadening based on filamentation in a gas-filled cell is an interesting alternative ${ }^{[9-12]}$ because of (i) wide and smooth output spectra that cover visible wavelength with an energy of $>1 \mu \mathrm{J}$, (ii) free-space nonlinear propagation that has spatial filtering effects ${ }^{[13]}$ as well as removing the beam pointing issue, (iii) a wide acceptance range of the input pulse duration up to $\sim 100$ fs and (iv) simplicity compared with the NOPA scheme. However, the short-wavelength components of white light produced by filamentation are weaker than the fundamental components around $800 \mathrm{~nm}$ by two orders of magnitude. Spectral selection and phase compensation are thus crucial. To solve these problems, we used (i) specially designed band-stop mirrors to block the fundamental wavelength in $720-850 \mathrm{~nm}$, (ii) commercially available broadband dielectric mirrors to select the spectral range in 530-700 $\mathrm{nm}$ and (iii) a pair of dispersion compensation mirrors to compress the spectrally filtered pulses down to $6.5 \mathrm{fs}$. 


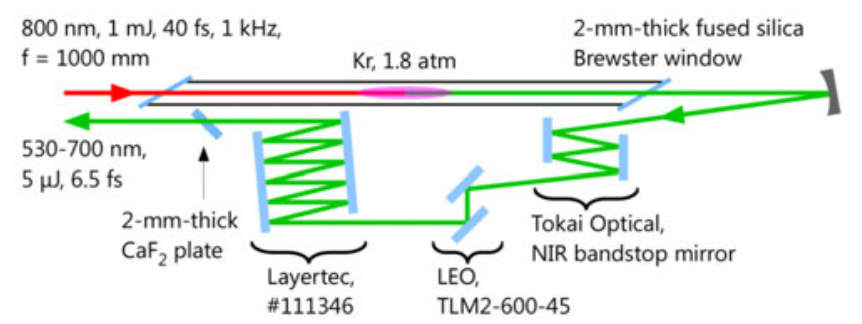

Figure 1. Schematic of the experimental setup. Note that the beams are all vertically polarized. The Brewster windows of the gas-filled cell are tilted vertically.

\section{Experiment}

\subsection{Filamentation and pulse compression}

Figure 1 shows the experimental setup of filamentation and pulse compression. The output pulses from a Ti:sapphire chirped-pulse amplification system (1 mJ, $40 \mathrm{fs}, 800 \mathrm{~nm}$ ) operated at $1 \mathrm{kHz}$ were loosely focused by a lens ( $f=$ $1000 \mathrm{~mm}$ ) to a 1-m-long gas cell filled with krypton at $1.8 \mathrm{~atm}$. The beam was focused at the center of the cell. Filamentation in the gas cell produced white light that covered from 350 to $2000 \mathrm{~nm}$. We did not observe shorter wavelength components below $350 \mathrm{~nm}$ because of the reflectivity cutoff of silver mirrors.

As shown in Figure 1, we used two kinds of dielectric mirrors for spectral selection and a pair of chirp mirrors for pulse compression. First, the pulses were reflected four times on two near-infrared band-stop mirrors (Tokai Optical Co., Ltd), which were specially designed to suppress the fundamental spectral components in 720-850 nm. Figures 2(a) and (b) show the measured reflectivity and group delay of the band-stop mirror, respectively. The reflectivity was measured with a spectrophotometer (JASCO Co., V570), and the group delay was measured by a home-built white-light interferometer ${ }^{[14]}$. The group delay dispersion is approximately $-30 \mathrm{fs}^{2}$ at $630 \mathrm{~nm}$. This mirror can efficiently block the fundamental components, while it does not affect the phase of the spectrally broadened components as shown in Figure 2(b).

This band-stop mirror was originally designed for difference frequency generation between the short and long wavelength components after spectral broadening ${ }^{[15]}$. Therefore, it has a high reflectivity range above and below the fundamental wavelength around $800 \mathrm{~nm}$. To separate the spectral components in the visible part around $600 \mathrm{~nm}$ from the infrared part reflected by the band-stop mirror, we used two dielectric mirrors (Lattice Electro Optics, Inc., TLM2-600-45). The incoming beam was s-polarized at these mirrors to maximize the reflected bandwidth. In total, four times of reflection on the band-stop mirrors and two times of reflection on the broadband dielectric mirrors were allowed us to select the visible part in 530-700 nm. Dispersion compensation was achieved by five bounces on each of a pair of chirped mirrors (Layertec GmbH, \#111346, approximately $-80 \mathrm{fs}^{2}$ per double bounces at $630 \mathrm{~nm}$ ). This chirped mirror pair can compensate the dispersion of 1.5-mm-thick fused silica or 2.1-mm-thick calcium fluoride $\left(\mathrm{CaF}_{2}\right)$ plates per one round trip. We used a $2-\mathrm{mm} \mathrm{CaF}_{2}$ plate to control the dispersion by changing the incident angle.

Figure 3 shows the dispersion of 1 -m-long air in the standard condition ${ }^{[16]}$ that is nearly identical to the dispersion of 0.5 -mm-thick fused silica ${ }^{[17]}$ or $0.7-\mathrm{mm}$-thick $\mathrm{CaF}_{2}{ }^{[18]}$. As can be seen in Figure 3, the dispersion of the air would significantly induce phase distortion if the propagation length exceeds $\sim 0.1 \mathrm{~m}$. Therefore, we optimized the dispersion of the visible pulses at the point of use by adjusting the number of reflections on the chirped mirrors as well as changing the effective thickness of a fused silica or $\mathrm{CaF}_{2}$ plate in the beam path.

The compressed pulses were characterized by the SHGbased frequency-resolved optical gating (FROG) ${ }^{[19,20]}$ that employed an $8-\mu$ m-thick BBO crystal. We obtained the
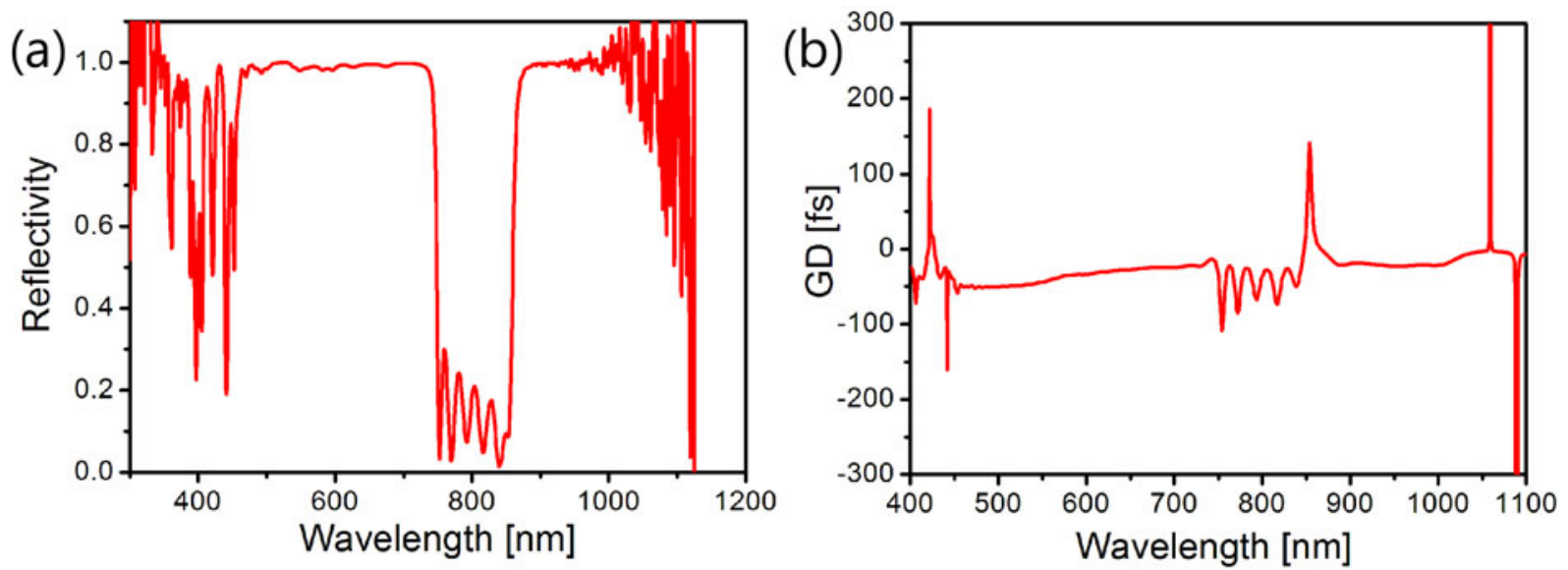

Figure 2. (a) Measured reflectivity and (b) group delay of the band-stop mirror. 


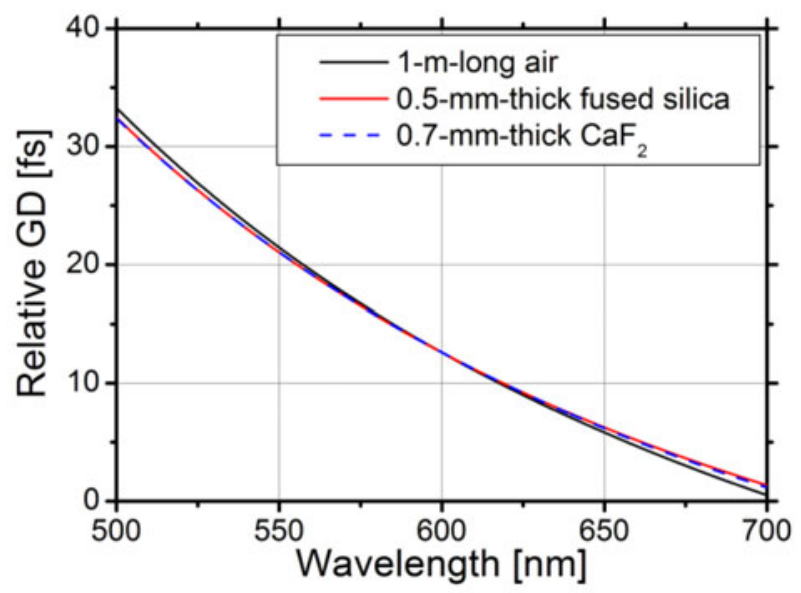

Figure 3. Relative group delays of 1-m-long air (black line), 0.5-mm-thick fused silica (red line) and 0.7-mm-thick $\mathrm{CaF}_{2}$ (blue dashed line).

nearly transform-limited 6.5 -fs pulses with a pulse energy of $5 \mu \mathrm{J}$ and a smooth spectrum in $530-700 \mathrm{~nm}$ as shown in Figure 4.
We inspected the beam quality and pointing stability after the pulse compression. Figures 5(a) and (b) show the beam profiles after pulse compression before and after focusing with a concave mirror $(f=300 \mathrm{~mm})$, respectively. Because of the spatial filtering effect in filamentation, both of the beam profiles were nearly Gaussian. The $1 / e^{2}$ intensity beam radii of the near-field beam profile were measured to be 936 and $862 \mu \mathrm{m}$ along the horizontal (X) and vertical (Y) directions, respectively, and those of the far-field beam profile were 79 and $82 \mu \mathrm{m}$. Pointing stability of the beam before focusing was measured by a quadrant-cell photodetector. The fluctuation of the beam center with respect to the $1 / e^{2}$ intensity beam radius was $\sim 5 \times 10^{-3}$.

\subsection{Spectral stability measurement}

We also measured the stability of the spectrum broadened by the filamentation process in a gas-filled cell since shotto-shot reproducibility is essential for various spectroscopic applications. We put a bandpass filter (Thorlabs, Inc., FESH0700) just behind the gas-filled cell to select the
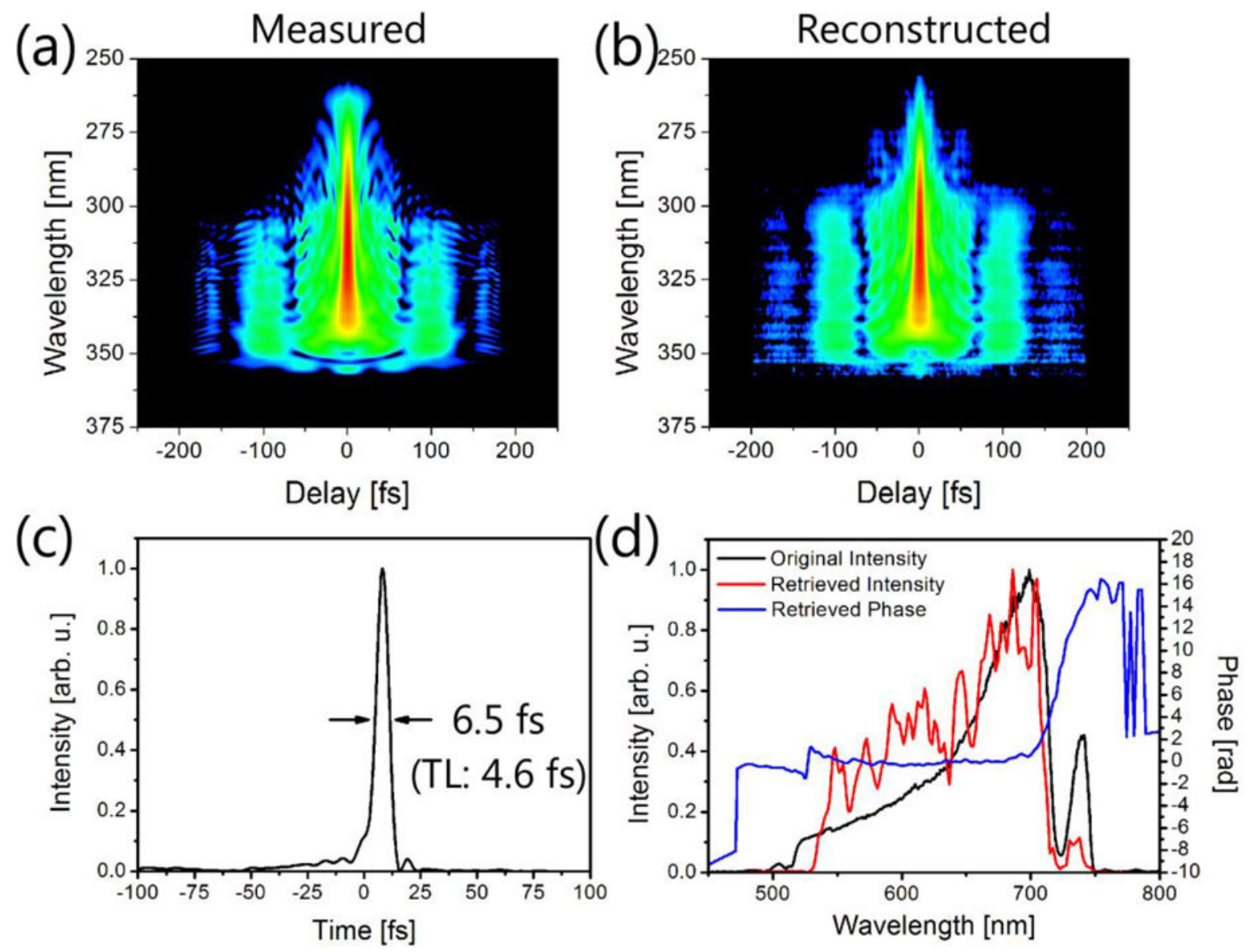

Figure 4. Results of SHG-FROG measurement. (a), (b) Measured and reconstructed FROG traces, respectively. (c) Retrieved temporal intensity profile (measured: $6.5 \mathrm{fs}$, transform-limited case: $4.6 \mathrm{fs}$ ), (d) Intensity spectrum measured by a spectrometer (black line), retrieved spectrum (red line), and retrieved spectral phase (blue line). 

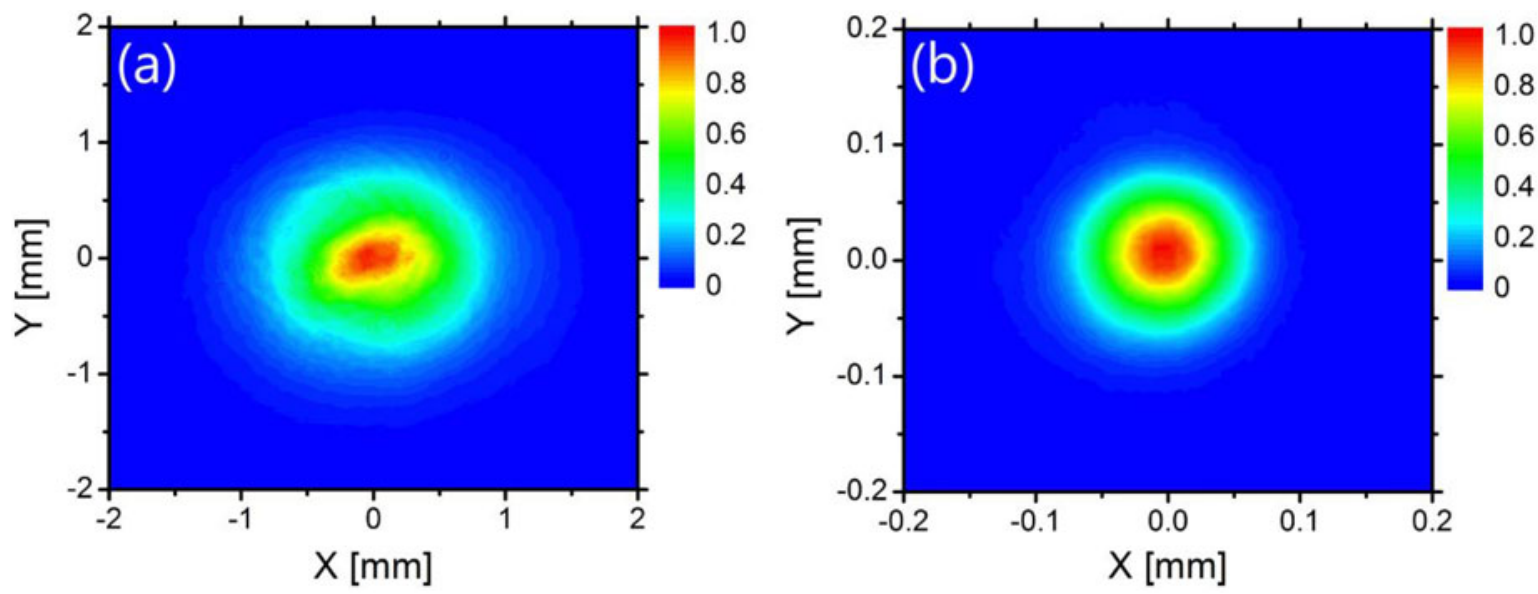

Figure 5. Beam profiles of the compressed pulse (a) before and (b) after focusing with a concave mirror $(f=300 \mathrm{~mm})$.
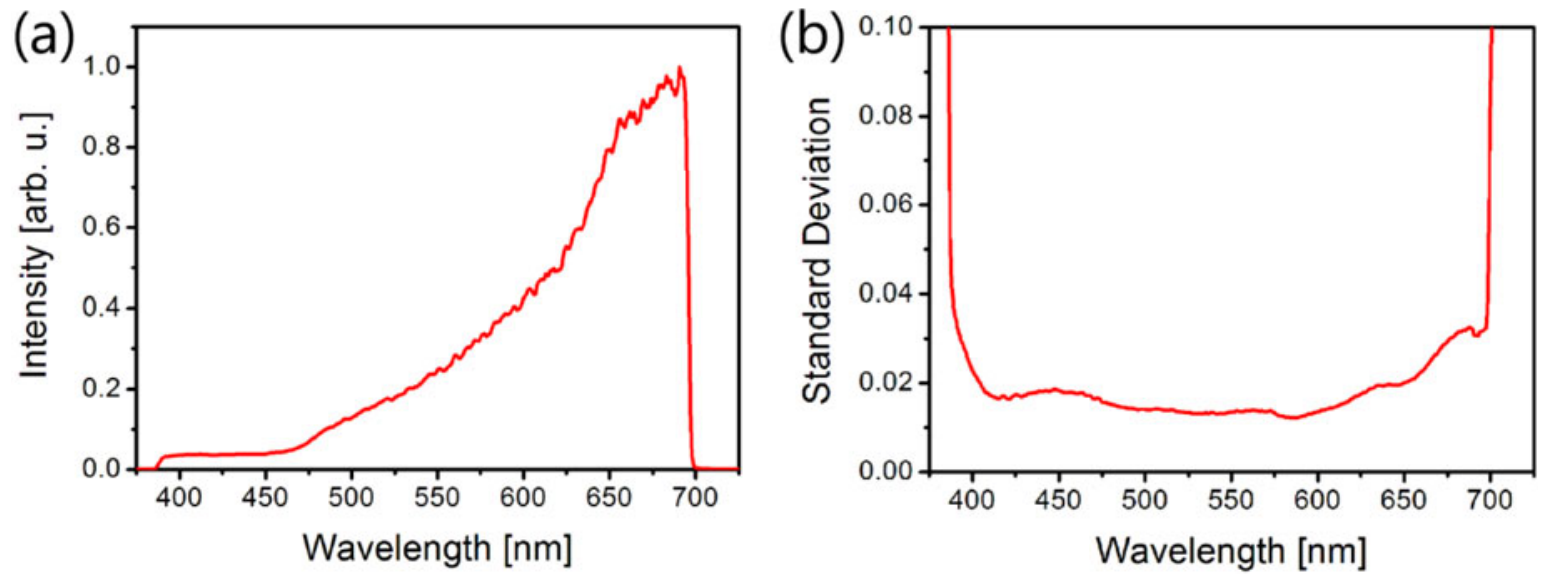

Figure 6. Results of the spectral stability measurement. (a) Averaged spectrum of 1000 single-shot spectra. (b) Standard deviation of each spectral component.

spectral components from 400 to $700 \mathrm{~nm}$, then measured single-shot spectra using a spectrometer (Ocean Optics, Inc., HR2000+). Figures 6(a) and (b) show the averaged spectrum over 1000 shots and the standard deviation of each spectral component, respectively. The standard deviation of the pulse energies before filamentation process was measured to be $1.97 \%$. Figure 6(b) shows that the spectral instability of the white light in $420-650 \mathrm{~nm}$ was $\sim 2 \%$, which is similar to the fluctuation of the input pulse energy. The shot-to-shot fluctuation in $650-750 \mathrm{~nm}$ is slightly higher than that of 420-650 nm, which was probably due to the SPM in the exit window of the cell. These results show that the white-light continuum produced by filamentation in a gasfilled cell is suitable for spectroscopic applications.

\section{Conclusion}

We have produced $5-\mu \mathrm{J}, 6.5$-fs visible pulses at a repetition rate of $1 \mathrm{kHz}$ using filamentation in a gas cell filled with krypton followed by spectral selection and phase compensation by a combination of dielectric mirrors. The shot-to-shot spectral instability of the visible continuum was measured to be $\sim 2 \%$ (standard deviation) in $420-650 \mathrm{~nm}$, which was almost the same as the energy fluctuation of the output pulses from our Ti:sapphire chirped-pulse amplifier. This pulse compression scheme is simple and robust, and can be easily integrated into intense ultrashort-pulse laser systems. This setup will be used for time-resolved soft-x-ray spectroscopy using high harmonics with sub-10-fs temporal resolution.

\section{Acknowledgments}

This research was partially supported by Grant-in-Aid for Scientific Research (S) Grant Number 23226003, Grant-inAid for Young Scientists (B) Grant Number 25790063 and Program for Leading Graduate Schools (MERIT) by Japan Society for the Promotion of Science. 


\section{References}

1. T. Popmintchev, M.-C. Chen, D. Popmintchev, P. Arpin, S. Brown, S. Ališauskas, G. Andriukaitis, T. Balčiunas, O. D. Mücke, A. Pugzlys, A. Baltuška, B. Shim, S. E. Schrauth, A. Gaeta, C. Hernández-García, L. Plaja, A. Becker, A. JaronBecker, M. M. Murnane, and H. C. Kapteyn, Science 336, 1287 (2012).

2. N. Ishii, K. Kaneshima, K. Kitano, T. Kanai, S. Watanabe, and J. Itatani, Nat. Commun. 5, 3331 (2014).

3. S. Cousin, F. Silva, S. Teichmann, M. Hemmer, B. Buades, and J. Biegert, Opt. Lett. 39, 5383 (2014).

4. S. Koshihara and S. Adachi, J. Phys. Soc. Japan 75, 011005 (2006).

5. G. Cerullo, M. Nisoli, S. Stagira, and S. De Silvestri, Opt. Lett. 23, 1283 (1998).

6. A. Shirakawa, I. Sakane, M. Takasaka, and T. Kobayashi, Appl. Phys. Lett. 74, 2268 (1999).

7. M. Nisoli, S. De Silvestri, and O. Svelto, Appl. Phys. Lett. 68, 2793 (1996).

8. Y. Matsubara, S. Ogihara, J. Itatani, N. Maeshima, K. Yonemitsu, T. Ishikawa, Y. Okimoto, S. Koshihara, T. Hiramatsu, Y. Nakano, H. Yamochi, G. Saito, and K. Onda, Phys. Rev. B 89, 161102(R) (2014).
9. P. B. Corkum, C. Rolland, and T. Srinivasan-Rao, Phys. Rev. Lett. 57, 2268 (1986).

10. A. Braun, G. Korn, X. Liu, D. Du, J. Squier, and G. Mourou, Opt. Lett. 20, 73 (1995).

11. C. P. Hauri, W. Kornelis, F. W. Helbing, A. Heinrich, A. Couairon, A. Mysyrowicz, J. Biegert, and U. Keller, Appl. Phys. B 79, 673 (2004).

12. L. Gallmann, T. Pfeifer, P. M. Nagel, M. J. Abel, D. M. Neumark, and S. R. Leone, Appl. Phys. B 86, 561 (2007).

13. B. Prade, M. Franco, A. Mysyrowicz, A. Couairon, H. Buersing, B. Eberle, M. Krenz, D. Seiffer, and O. Vasseur, Opt. Lett. 31, 2601 (2006).

14. L. Lepetit, G. Chériaux, and M. Joffre, J. Opt. Soc. Am. B 12, 2467 (1995)

15. N. Ishii, K. Kaneshima, K. Kitano, T. Kanai, S. Watanabe, and J. Itatani, Opt. Lett. 37, 4182 (2012).

16. P. E. Ciddor, Appl. Opt. 35, 1566 (1996).

17. I. H. Malitson, J. Opt. Soc. Am. 55, 1205 (1965).

18. H. H. Li, J. Phys. Chem. Ref. Data 9, 161 (1980).

19. K. W. DeLong, R. Trebino, J. Hunter, and W. E. White, J. Opt. Soc. Am. B 11, 2206 (1994).

20. A. Baltuska, M. S. Pshenichnikov, and D. A. Wiersma, IEEE J. Quant. Electron. 35, 459 (1999). 